\title{
Microstructure of the Default Mode Network in Preterm Infants
}

\author{
(D). Cui, (D) Tymofiyeva, (D) R. Desikan, (D)T. Flynn, (D) H. Kim, (DD. Gano, (D) C.P. Hess, (DD.M. Ferriero, (D)A.J. Barkovich, and DD. Xu
}

\begin{abstract}
BACKGROUND AND PURPOSE: Diffusion and fMRI has been providing insights to brain development in addition to anatomic imaging. This study aimed to evaluate the microstructure of white matter tracts underlying the default mode network in premature infants by using restingstate functional MR imaging in conjunction with diffusion tensor imaging-based tractography.
\end{abstract}

MATERIALS AND METHODS: A cohort of 44 preterm infants underwent structural T1-weighted imaging, resting-state fMRI, and DTI at 3T, including 21 infants with brain injuries and 23 infants with normal-appearing structural imaging as controls. Neurodevelopment was evaluated with the Bayley Scales of Infant Development at 12 months' adjusted age. Probabilistic independent component analysis was applied to resting-state fMRI data to explore resting-state networks. The localized clusters of the default mode network were used as seeding for probabilistic tractography. The DTI metrics (fractional anisotropy, mean diffusivity, axial diffusivity, and radial diffusivity) of the reconstructed primary tracts within the default mode network-cingula were measured.

RESULTS: Results revealed decreased fractional anisotropy $(0.20 \pm 0.03)$ and elevated radial diffusivity values $(1.24 \pm 0.16)$ of the cingula in the preterm infants with brain injuries compared with controls (fractional anisotropy, $0.25 \pm 0.03 ; P<.001$; radial diffusivity, $1.06 \pm 0.16$; $P=.001)$. The Bayley Scales of Infant Development cognitive scores were significantly associated with cingulate fractional anisotropy $(P=$ $.004)$ and radial diffusivity $(P=.021)$; this association suggests that the microstructural properties of interconnecting axonal pathways within the default mode network are of critical importance in the early neurocognitive development of infants.

CONCLUSIONS: This study of combined resting-state fMRI and DTI at rest suggests that such studies may allow the investigation of key functional brain circuits in premature infants, which could function not only as diagnostic tools but also as biomarkers for long-term neurodevelopmental outcomes.

ABBREVIATIONS: $A D=$ axial diffusivity; $B I=$ brain injuries; BSID-III = Bayley Scales of Infant Development; $D M N=$ default mode network; $F A=$ fractional anisotropy; $\mathrm{GA}$ = gestational age; $\mathrm{IVH}$ = intraventricular hemorrhage; $\mathrm{MD}=$ mean diffusivity; $\mathrm{RD}=$ radial diffusivity; $\mathrm{RSNs}$ = resting-state networks; $\mathrm{WMI}=$ white matter injury

$\mathbf{P}$ rematurely born infants face heightened risks of brain injuries, such as intraventricular hemorrhage (IVH) and white matter injury (WMI). The brain injuries may disrupt normal cerebral development and result in long-term neurodevelopmental disabilities, including cognitive impairment, behavioral prob-

Received March 9, 2016; accepted after revision September 6.

From the Departments of Radiology and Biomedical Imaging (J.C., O.T., R.D., T.F., H.K., C.P.H., A.J.B., D.X.) and Pediatrics and Neurology (D.G., D.M.F.), University of California, San Francisco, San Francisco, California.

This work was supported by National Institutes of Health grants R01EB009756, R01HD072074, R01NS046432, R21AT009173, and P01NS082330.

Please address correspondence to Duan Xu, PhD, 1700 4th St, BH102, UCSF Box 2512, San Francisco, CA 94143-2512; e-mail: Duan.Xu@ucsf.edu

- Indicates open access to non-subscribers at www.ajnr.org

$\equiv$ Indicates article with supplemental on-line tables.

Indicates article with supplemental on-line photo.

http://dx.doi.org/10.3174/ajnr.A4997 lems, and psychiatric alterations. ${ }^{1-3}$ A brain neural network is a series of interconnected neurons whose information is continuously processed and transported between structurally and functionally linked brain regions. ${ }^{4}$ The maturation of neural networks plays an important role in cortical development because neural activity is essential for refining and shaping the intricate circuitry of the nervous system. ${ }^{5}$ Recently, a growing number of studies have used noninvasive resting-state fMRI to characterize these networks during brain development. .-11 $^{-11}$

Resting-state fMRI enables the detection of spontaneous spatially coherent fluctuations of the blood oxygen level-dependent signals at rest. ${ }^{12}$ Application of resting-state fMRI has led to the identification of the resting-state networks (RSNs) encompassing regions of the brain involved in attention, language, behavior, and cognitive function. ${ }^{13,14}$ Resting-state fMRI is a promising technique for the study of cerebral development in infants because no specific task performance or cognitive ability is required..$^{7-11,15}$ 
Several recent studies have observed the emergence of RSNs in the brains of preterm infants, noting that visual, auditory, somatosensory, motor, default mode, frontoparietal, and executive control networks develop at different rates. ${ }^{7-11,16}$ Among the RSNs, the most widely studied is the default mode network (DMN), which is associated with cognitive development. ${ }^{17-19}$ In adults, the DMN consists of the medial prefrontal cortex, the posterior cingulate cortex/precuneus, the inferior parietal cortex, and their associated connections. Functional "connectivity" between the main nodes is supported by an underlying structure of white matter pathways, with the cingulum as the key tract that interconnects the anterior and posterior core regions of the DMN. ${ }^{20}$ Damage to the cingulum may result in a broad range of brain disorders. ${ }^{17-19}$ Until now, little was known about the characteristics of the structural pathways underlying the DMN in premature infants. Here, we implemented resting-state $\mathrm{fMRI}$, combined with diffusion tensor imaging-based tractography, to characterize the white matter pathways within the premature infant DMN.

DTI assesses random motion of water molecules within biologic tissue and provides insight into microstructure. ${ }^{21,22}$ In the tensor model, which has 3 eigenvalues that represent the magnitude of diffusion in 3 orthogonal axis directions, ${ }^{21}$ fractional anisotropy (FA) is calculated from the variance of the 3 eigenvalues to indicate the degree of anisotropy. ${ }^{23}$ Mean diffusivity (MD) is the average of the 3 eigenvalues and a scalar measure of the total diffusion. ${ }^{24}$ The eigenvector corresponding to the maximum diffusivity is assumed to be directed parallel to the largest group of white matter fibers. Its eigenvalue is referred to as axial diffusivity (AD). The secondary and tertiary eigenvectors oriented perpendicular to the primary eigenvector and the average of their eigenvalues are referred to as "radial diffusivity" (RD). The aims of the present study were the following: 1) to determine the structural pathway of the DMN in these 2 groups of infants (injured and control) and to compare the DTI measures between them; and 2) to further investigate their correlation with longitudinal brain neurocognitive development. We hypothesized that significantly different diffusion parameters would be found in infants with brain injuries compared with normally developing infants. It was also hypothesized that the microstructural architecture inferred by water diffusion of the cingula would be associated with cognitive functioning.

\section{MATERIALS AND METHODS \\ Subjects}

Fifty-one preterm infants, 25 males and 26 females, born prematurely between 24.7 and 32.3 gestational weeks (mean, $28.8 \pm 1.8$ weeks) underwent MR imaging at 29.8 35.6 gestational weeks $(32.2 \pm 1.5$ weeks $)$. All datasets were visually inspected, and 7 were excluded due to the severe motion artifacts. The datasets of the remaining 44 infants who had a complete and successful (no signal loss or severe artifacts) MR imaging session were included. Radiologic assessments were completed by neuroradiologists experienced in neonatal imaging, including the IVH and WMI scoring systems as follows: IVH score: 0 , absent; 1 , germinal matrix hemorrhage; 2 , intraventricular hemorrhage, volume of blood of $<50 \%$ of the ventricular volume; 3 , intraventricular hemorrhage, volume of blood of $>50 \%$ of the ventricular volume; and 4 , periventricular hemorrhagic infarction. WMI scores were the following: 0, absent; 1 , minimal, up to 3 foci of WMI, each $<2 \mathrm{~mm}$; 2 , moderate, $>3$ foci of WMI of $<2 \mathrm{~mm}$ or any foci of $>2 \mathrm{~mm}$; and 3 , severe, $>5 \%$ of hemisphere involved. The infants were divided into 2 groups: 21 infants with brain injuries (BI) (13 males and 8 females; gestational age [GA], $29.1 \pm 1.9$ weeks $)$ and 23 infants with normal-appearing brains ( 13 males and 10 females; GA, $29.1 \pm 1.5$ weeks); the latter were controls. A subset of participants (13 of 44 infants) had 12-month follow-up neurodevelopmental studies, and the developmental outcome was evaluated with the Bayley Scales of Infant Development (BSIDIII), which has separate composite scores for cognitive, motor, and language skills. The study was approved by the institutional review board, and written inform consent was given by the infants' parents.

\section{MR Imaging Acquisition}

Scans were obtained by using a 3T GE MR750 scanner (GE Healthcare, Milwaukee, Wisconsin). Structural 3D sagittal T1 inversion recovery-prepared fast-spoiled gradient-echo images were obtained with the following parameters: flip angle $=15^{\circ}$, FOV $=18 \mathrm{~cm}$, and voxel size $=1 \times 1 \times 1 \mathrm{~mm}$. T2*-weighed functional MR imaging scans were collected with a gradient-echo pulse sequence $\left(\mathrm{TR} / \mathrm{TE} / \mathrm{flip}\right.$ angle $=2 \mathrm{~s} / 20 \mathrm{~ms} / 90^{\circ}, \mathrm{FOV}=24 \mathrm{~cm}$, voxel size $=4 \times 4 \times 4 \mathrm{~mm}$, and no gap). Whole-brain DTI was acquired with a spin-echo echo-planar imaging diffusion sequence $(\mathrm{FOV}=25.6 \mathrm{~cm}$, acquisition matrix $=128 \times 128, \mathrm{TR} /$ $\mathrm{TE}=5 \mathrm{~s}$ /minimum, voxel size $=2 \times 2 \times 2 \mathrm{~mm}$ ). Diffusion gradients were applied in 30 noncollinear and noncoplanar directions with $b=600 \mathrm{~s} / \mathrm{mm}^{2}$.

\section{Functional Image Processing}

Data processing was performed by using FSL, Release 5.0 (http:// www.fmrib.ox.ac.uk/fsl). Preprocessing procedures included the following: motion correction with MCFLIRT (http://fsl.fmrib.ox. ac.uk/fsl/fslwiki/MCFLIRT) ${ }^{25}$; section-timing correction; brain extraction with the FSL Brain Extraction Tool (BET; http://fsl. fmrib.ox.ac.uk/fsl/fslwiki/BET) ${ }^{26}$; spatial smoothing with a Gaussian kernel of full width at half maximum of $6 \mathrm{~mm}$; and high-pass temporal filtering with 100 seconds. Functional MR imaging data were aligned to the T1-weighted image with $12 d f$. The structural scan was aligned with the Montreal Neurological Institute pediatric atlas (http://www.bic.mni.mcgill.ca/ServicesAtlases/NIHPDobj2) by using nonlinear registration. Transformation of the functional results into Montreal Neurological Institute space was performed following concatenation of the 2 alignments into a single matrix. All spatially normalized fMRI data were resampled to $1-\mathrm{mm}^{3}$ resolution. FSL Multivariate Exploratory Linear Decomposition into Independent Components (MELODIC; http://fsl.fmrib.ox.ac.uk/fsl/fslwiki/MELODIC ${ }^{27}$ ) was used to perform an independent component analysis on the fMRI data of all the subjects $(n=44)$ and to automatically estimate the dimensions of the independent components.

\section{Diffusion Image Processing}

DTI data were processed with the FMRIB Diffusion Toolbox (FDT; http://fsl.fmrib.ox.ac.uk/fsl/fslwiki/FDT). ${ }^{28} \mathrm{~A}$ standard 


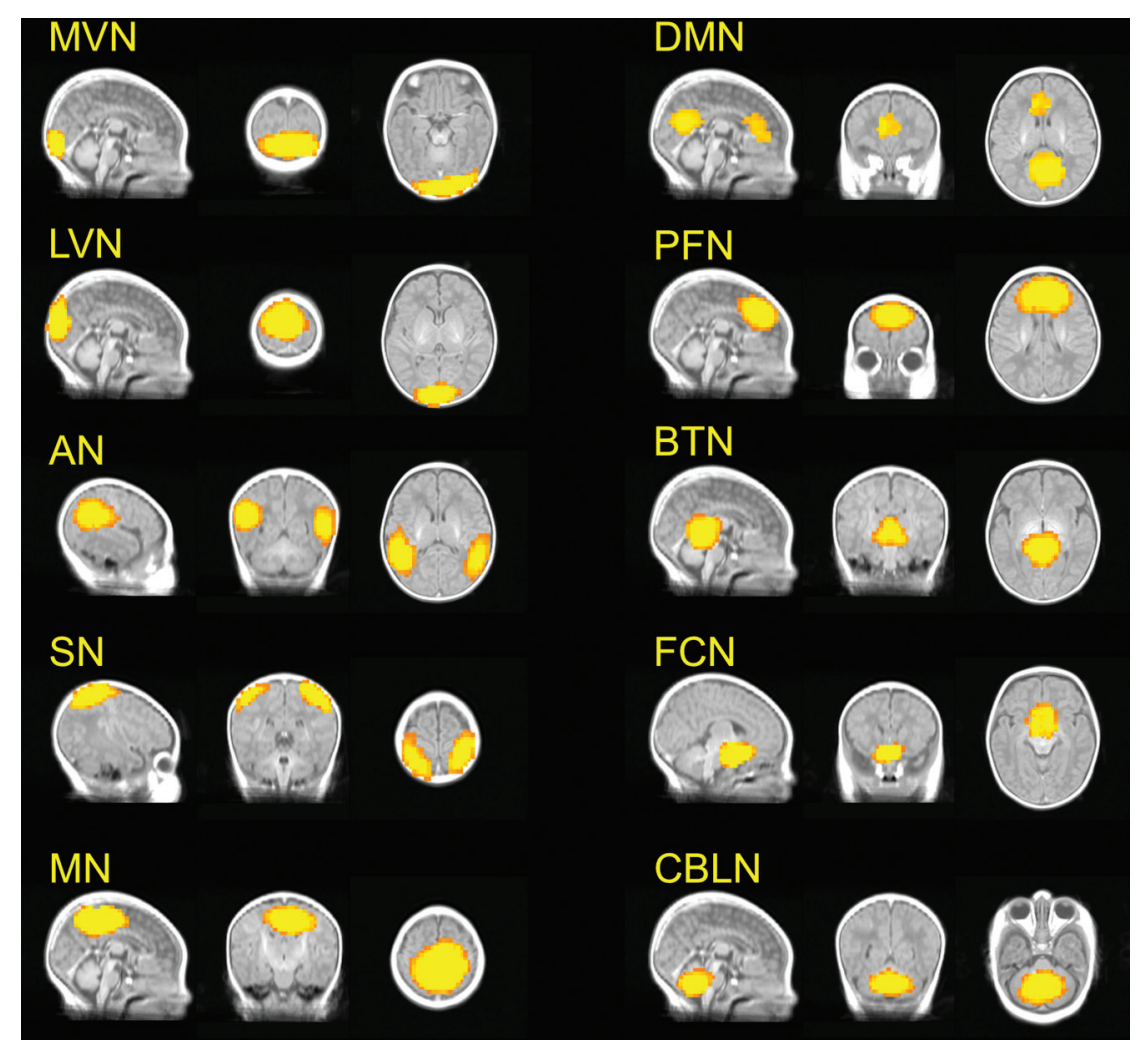

FIG 1. RSNs $(z>2.6)$ identified by group independent component analysis in preterm infants $(n=$ 44). MVN indicates medial visual network; LVN, lateral visual network; $A N$, auditory network; SN, salience network; MN, motor network; PFN, prefrontal network; BTN, brain stem and thalami; FCN, frontal cortical network; and CBLN, cerebellum network.

FDT multistep procedure was performed, including the following: 1) image-quality check (any gradient directions with signal dropouts caused by excessive motion were removed and not included in the analysis ${ }^{29}$ ); 2) motion and eddy current correction; 3) correction of gradient directions for any image rotation during the previous motion and eddy current correction; 4) removal of skull and nonbrain tissue by using the BET; 5) tensor reconstruction by using a weighted least-squares fit was performed via DTIFit (http://fsl.fmrib.ox.ac.uk/fsl/fsl-4.1.9/fdt/fdt_dtifit.html) within FDT to create DTI scalar images, including FA, MD, and 3 eigenvalues. The $\mathrm{RD}$ maps were computed as the average of the 2 minor eigenvalue images. The $\mathrm{MD}, \mathrm{AD}$, and $\mathrm{RD}$ values were reported in square millimeters/second $\times 10^{-3}$.

\section{Probabilistic Tractography}

Fiber tracking was performed by using ProbtrackX (http://fsl.fmrib.ox. ac.uk/fsl/fslwiki/FDT/UserGuide\#PROBTRACKX_-_probabilistic_ tracking_with_crossing_fibres) in FSL. ${ }^{30}$ First, diffusion parameters were estimated at each voxel by using BedpostX (http://fsl.fmrib.ox. ac.uk/fsl/fslwiki/FDT/UserGuide\#BEDPOSTX). ProbtrackX was then used to estimate the distribution of connections with 5000 streamline samples, a step length of $0.5 \mathrm{~mm}$, and a curvature threshold of 0.2. We used the localized regions of the DMN $(z>$ $2.6)$ in the resting-state fMRI analysis as seed regions. Seed regions were transformed from standard space (Montreal Neurological Institute pediatric $1-\mathrm{mm}^{3}$ standard brain) to each individual diffusion space by using a nonlinear transform (standard2diff) with $12 d f$. Generated pathways are volumes in which values at each voxel represent the number of streamlines passing through that voxel and, therefore, the probability of a connection between paired seed regions. To remove background noise, we thresholded pathways in each individual to include only voxels with at least 100 samples passing through them (of 5000 initiated streamline samples). ${ }^{31}$ Pathways in each subject were binarized and overlaid to produce population probability maps for each pathway, in which voxel values represent the number of subjects in whom a pathway is present. The population probability maps were thresholded at $50 \%$ of the maximum number of participants who had overlapping connections between paired seed regions to generate a group tract map. ${ }^{32,33}$ The obtained group tract map was then transformed to individual diffusion space and binarized to mask the FA, $\mathrm{MD}, \mathrm{AD}$, and $\mathrm{RD}$ maps for DTI metrics measurement.

\section{Statistical Analysis}

Statistical analysis was performed by using SPSS Statistics 22 (IBM, Armonk, New York). The Kolmogorov-Smirnov test of normality was used to confirm the normal distribution of BSID-III scores and DTI metrics $(P>.05)$. A 2-tailed independent-samples $t$ test was used to compare the birth GA, postmenstrual age at scan, and birth weights between the 2 groups. General linear regression was performed to compare the DTI metrics between groups, correcting for the birth GA, postmenstrual age at scan, sex, and birth weights. To investigate the association between the DTI metrics and BSID-III cognitive scores, we performed partial correlations; and the birth GA, postmenstrual age at scan, sex, and birth weights were also included as nuisance covariates.

\section{RESULTS}

\section{Demographics}

The 2-tailed independent-samples $t$ test demonstrated that there was no significant difference in birth GA (BI, $29.1 \pm 1.9$ weeks; control, 29.1 \pm 1.6 weeks; $P=.96)$, GA at scanning (BI, $32.4 \pm 1.6$ weeks; control, $32.2 \pm 1.5$ weeks; $P=.76$ ), and birth weights (BI, $1278 \pm 290$ g; control, $1241 \pm 273 \mathrm{~g} ; P=.65$ ) between groups. The detailed demographic information of the infants is summarized in On-line Table 1.

\section{RSNs}

Forty-three independent components were obtained from group independent component analysis decomposition across all the subjects $(n=44)$. RSNs were identified on the basis of the location and the frequency spectrum. Ten known RSNs were selected (Fig 1), including the medial visual network, lateral visual network, auditory network, salience network, motor network, DMN, 


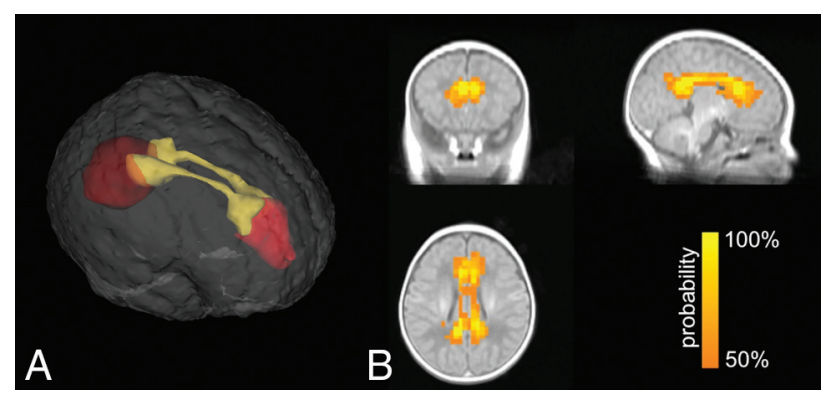

FIG 2. Group tract map of the reconstructed cingula. Each voxel value represents the probability that subjects had some cingula in it.
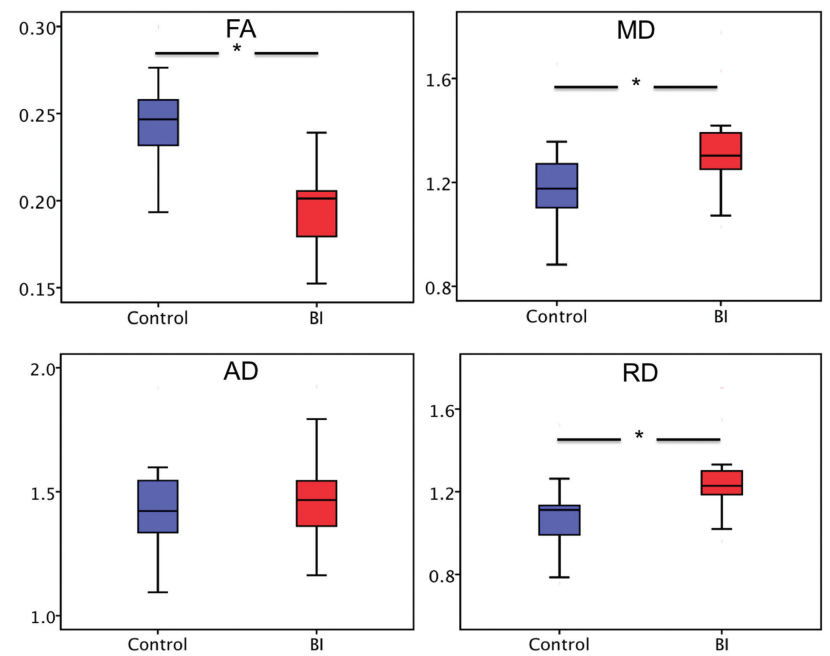

FIG 3. Boxplots illustrate the group differences in DTI metrics. The asterisk indicates $P<.05$.

prefrontal network, brain stem and thalami network, frontal cortical network, and cerebellum network. Fourteen additional RSNs were found as the subnetworks of these 10 RSNs, such as the visual network and prefrontal network as well as the subcomponents of some known RSNs, such as the frontoparietal network and executive control network (On-line Figure). Only noise or artifacts were found in the remaining 19 RSNs. The RSN of interest in the current study is the DMN. As shown in Fig 1, the DMN generated from the group independent component analysis on 44 preterm infants contains the regions of the medial prefrontal cortex (cluster size $=162$ voxels) and posterior cingulate cortex/precuneus (411 voxels).

\section{Group Comparison in DTI Metrics of the Reconstructed Cingula}

As shown in Fig 2, the bilateral cingulum bundles, which are the primary fiber tracts connecting the medial prefrontal cortex and the posterior cingulate cortex/precuneus, were reconstructed. Figure 3 shows the group comparison in DTI metrics of the reconstructed cingula. The FA values in the BI group $(0.20 \pm 0.03)$ were significantly lower than those in the control group $(0.25 \pm$ $0.03, F=26.79, P<.001)$. The MD and RD values were both elevated in the $\mathrm{BI}$ group $(\mathrm{MD}, 1.32 \pm 0.17 ; \mathrm{RD}, 1.24 \pm 0.16)$ compared with the control group $(\mathrm{MD}, 1.19 \pm 0.16 ; F=6.44, P=$ $.015 ; \mathrm{RD}, 1.06 \pm 0.16 ; F=13.35, P=.001)$. No significant difference was found in $\mathrm{AD}$ between the 2 groups (BI, $1.47 \pm 0.19$; control, $1.44 \pm 0.18 ; F=0.12, P=.731)$.
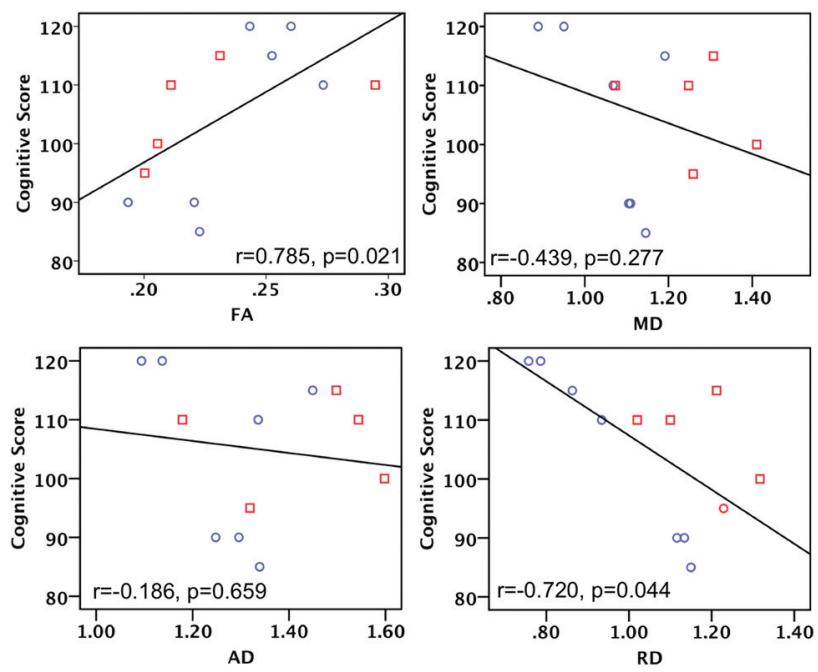

FIG 4. Scatterplots illustrate the correlation between BSID-III cognitive scores and DTI metrics in 13 infants, including 6 with brain injuries (red rectangle) and 7 controls (blue circle). It was found that the cognitive score is positively associated with FA and negatively associated with RD.

\section{Association between DTI Metrics and BSID-III Scores}

As shown in Fig 4, the cognitive scores were positively correlated with the FA values $(r=0.848, P=.004)$ and negatively correlated with the RD values $(r=-0.746, P=.021)$. In addition to cognitive scores, we also examined the association between the DTI metrics and the language and motor scores. Language scores were positively associated with the FA values $(r=0.782, P=.013)$ but not significantly correlated with the $\mathrm{MD}, \mathrm{AD}$, or $\mathrm{RD}$ values. The motor scores were not correlated with the DTI metrics (On-line Table 2).

\section{DISCUSSION}

In the present study, resting-state fMRI was used to identify key RSNs and localize the medial prefrontal cortex and posterior cingulate cortex/precuneus within the DMN in prematurely born infants. We found that the DTI metrics within the cingula, the primary fiber tracts connecting the medial prefrontal cortex and posterior cingulate cortex/precuneus, were altered in the preterm infants with brain injuries. DTI abnormalities within the cingula were also significantly associated with decreased cognitive scores at 12 months of age. Our findings support the hypothesis that microstructural abnormalities of the DMN are present in preterm infants with brain injuries and are associated with impaired neurocognitive development. To the best of our knowledge, this is the first investigation of the microstructure of a specific neural network relevant to the neurocognitive development in preterm infants.

In 2007, Fransson et $\mathrm{al}^{10}$ performed the first study of RSN development in 12 preterm infants at 41 weeks' postmenstrual age. A presumed precursor of the DMN was observed in Fransson et al. ${ }^{8,9} \mathrm{Gao}$ et al ${ }^{11}$ explored the temporal and spatial development within the DMN and observed a primitive and incomplete DMN in 2-week-old neonates. Doria et $\mathrm{al}^{7}$ performed a longitudinal investigation to explore early development of RSNs from 29 weeks' postmenstrual age to term-equivalent age in 62 preterm infants. In line with the previous studies, the main architecture of the DMN, including the medial prefrontal cortex and posterior 
cingulate cortex/precuneus, was observed in our study, while other regions that are commonly observed in the DMNs of adults were not found, such as inferior parietal cortex, lateral temporal cortex, and hippocampal regions. Emerging evidence suggests that the posterior cingulate cortex and medial prefrontal cortex are consistently observed in the DMN and serve as 2 main "hubs" involved in different aspects of cognitive function. ${ }^{11,34}$ The medial prefrontal cortex might be involved in self-referential activity, mentalizing processes, and theory of mind, while the posterior cingulate cortex is more associated with implicit memory. ${ }^{35}$ As the structural foundation of the functional connectivity between these 2 hubs, the underlying white matter tracts, therefore, are vital to the neural signal transmission between the medial prefrontal cortex and posterior cingulate cortex.

By combining probabilistic independent component analysis and probabilistic tract reconstruction, we provide in vivo evidence that the microstructural architecture of the primary white matter tract within the DMN-cingula is disrupted in preterm infants with brain injuries. The BI group showed significantly lower FA and elevated MD and RD values compared with the control group. This change in DTI metrics of the white matter was consistent with the previous DTI studies in preterm infants. ${ }^{15,36-38}$ Counsell et $\mathrm{al}^{36}$ found that the apparent diffusion coefficient values were significantly higher in the infants with white matter injury than that in the infants with normal-appearing white matter in the frontal, central, and posterior white matter at the level of the centrum semiovale in 50 preterm infants at term-equivalent age. Thompson et $\mathrm{al}^{37}$ scanned 116 infants and found that perinatal white matter abnormality and IVH were associated with increased diffusivity in the white matter of very preterm infants. Very recently, Morita et $\mathrm{al}^{38}$ evaluated the cerebellar and cerebral white matter of 42 preterm infants and found that preterm infants with IVH had lower FA values compared with infants without IVH. Until now, no studies have assessed the microstructure of the cingulum bundles in the preterm infant with brain injuries, to our knowledge. Our results first revealed the altered FA, MD, and RD values of the cingula in the BI group compared with the normalappearing preterm infants. In preterm infants, low FA likely indicates low axonal fiber density, delayed premyelination, or increased water content. In our study, increases in $\mathrm{RD}$ with little change in $\mathrm{AD}$ were observed in the preterm infants with brain injuries. This finding suggests that the changes in anisotropy are not simply explained by changes in brain-water content and more likely reflect the microstructural changes, causing decreased hindrance to water diffusion perpendicular to the direction of axonal fibers.

Within the cingula, we found that higher FA was associated with decreased cognitive abilities, and lower RD correlated with better cognitive and language scores of infants at 12 months of age. This finding is in accordance with the previous longitudinal studies in preterm infant neurodevelopment. ${ }^{37,39}$ van Kooij et $\mathrm{al}^{40}$ performed Tract-Based Spatial Statistics (TBSS; http://fsl. fmrib.ox.ac.uk/fsl/fslwiki/TBSS) analysis on 63 preterm infants and found that the FA in the corpus callosum at term-equivalent age was positively correlated with cognitive outcome (measured by the BSID-III) at 2-year corrected age. Thompson et $\mathrm{al}^{37}$ found that higher RD was associated with increased risk of impairment in motor and executive functions at 7 years of age in premature- born children. Currently, the results of our study demonstrate a correlation between cognitive performance and the cingula white matter microstructure measured at an early and critical phase of neurologic development. Previous studies have reported diffusivity decreasing and anisotropy increasing with increasing $\mathrm{GA},{ }^{41-43}$ and they are, therefore, signs of physiologic maturation. Indeed, these trends of diffusion metrics with progressive white matter maturation have been shown to continue throughout infancy and childhood. Using partial correlation to exclude the effects of aging, our study reveals that microstructural changes or delayed maturation in the developing white matter of the cingula might result in long-term neurocognitive impairment.

Several limitations to the present study should be considered. First, we did not delineate the anatomic representations of RSNs in the BI or control group separately. Preliminary results have demonstrated significant differences in RSN development in preterm infants with common forms of neuropathology. ${ }^{44}$ In a future study, we will apply independent component analysis with dual regression to resting-state fMRI data to investigate the altered brain circuits in preterm infants with brain injuries. Second, we focused on the DMN and its underlying primary white matter tract-cingulum bundles in this study. Other brain regions and neuronal tracts may be associated with neurocognitive development in infants. Nonhypothesized DTI analysis, such as TBSS, would be helpful to investigate the specificity of this effect. Third, the sample size ( $n=13$, including 6 with brain injuries) of our follow-up study was small. We are continuing to enroll additional subjects in the longitudinal study to explore the association between DTI metrics and neurodevelopment outcome for each group. Last, there may be microhemorrhages that may influence the diffusion measurements; however, as indicated by the results, the $\mathrm{MD}$ in the $\mathrm{BI}$ group is elevated in comparison with the control group; this finding suggests that susceptibility effects from microbleeds are small.

\section{CONCLUSIONS}

This study employed a multimodal imaging approach to evaluate the microstructural architecture of the DMN in preterm infants. Our results provide evidence that the microstructure of the primary white matter tracts connecting the medial prefrontal cortex and the posterior cingulate cortex/precuneus within the DMNcingula were disrupted in the preterm infants with brain injuries. Our findings reveal that higher degree of microstructural architecture of the cingula is associated with better neurocognitive abilities at 12 months, which suggests that the microstructural properties of the interconnecting axonal pathways between the 2 main hubs within the DMN play a critical role in the infants' neurodevelopment.

Disclosures: Olga Tymofiyeva-RELATED: Grant: National Institutes of Health.* Christopher P. Hess—RELATED: Grant: National Institutes of Health*; UNRELATED: Expert Testimony: medicolegal; Grants/Grants Pending: GE Healthcare, Quest Diagnostics, Cerebrotech*; Travel/Accommodations/Meeting Expenses Unrelated to Activities Listed: Siemens. Donna M. Ferriero—RELATED: Grant: National Institutes of Health*; Support for Travel to Meetings for the Study or other Purposes: National Institutes of Health*; UNRELATED: Grants/Grants Pending: National Institutes of Health*; Royalties: Elsevier. Anthony J. Barkovich—RELATED: Grant: National Institutes of Health. * Duan Xu—RELATED: Grant: National Institutes of Health. * *Money paid to the institution.

AJNR Am J Neuroradiol 38:343-48 Feb 2017 www.ajnr.org 


\section{REFERENCES}

1. Panigrahy A, Wisnowski JL, Furtado A, et al. Neuroimaging biomarkers of preterm brain injury: toward developing the preterm connectome. Pediatr Radiol 2012;42(suppl 1):S33-61 CrossRef Medline

2. Douglas-Escobar M, Weiss MD. Biomarkers of brain injury in the premature infant. Front Neurol 2012;3:185 CrossRef Medline

3. Miller SP, Cozzio CC, Goldstein RB, et al. Comparing the diagnosis of white matter injury in premature newborns with serial MR imaging and transfontanel ultrasonography findings. AJNR Am J Neuroradiol 2003;24:1661-69 Medline

4. Amit DJ. Modeling Brain Function: The World of Attractor Networks. New York: Cambridge University Press; 1989

5. Penn AA, Shatz CJ. Brain waves and brain wiring: the role of endogenous and sensory-driven neural activity in development. Pediatr Res 1999;45:447-58 CrossRef Medline

6. Smyser CD, Snyder AZ, Neil JJ. Functional connectivity MRI in infants: exploration of the functional organization of the developing brain. Neuroimage 2011;56:1437-52 CrossRef Medline

7. Doria V, Beckmann CF, Arichi T, et al. Emergence of resting state networks in the preterm human brain. Proc Natl Acad Sci U S A 2010;107:20015-20 CrossRef Medline

8. Fransson $\mathrm{P}$, Aden $\mathrm{U}$, Blennow $\mathrm{M}$, et al. The functional architecture of the infant brain as revealed by resting-state fMRI. Cereb Cortex 2011;21:145-54 CrossRef Medline

9. Fransson P, Skiöld B, Engström M, et al. Spontaneous brain activity in the newborn brain during natural sleep-an fMRI study in infants born at full term. Pediatr Res 2009;66:301-05 CrossRef Medline

10. Fransson $P$, Skiöld $B$, Horsch $S$, et al. Resting-state networks in the infant brain. Proc Natl Acad Sci U S A 2007;104:15531-36 CrossRef Medline

11. Gao W, Zhu H, Giovanello KS, et al. Evidence on the emergence of the brain's default network from 2-week-old to 2-year-old healthy pediatric subjects. Proc Natl Acad Sci U S A 2009;106:6790-95 CrossRef Medline

12. Biswal B, Yetkin FZ, Haughton VM, et al. Functional connectivity in the motor cortex of resting human brain using echo-planar MRI. Magn Reson Med 1995;34:537-41 CrossRef Medline

13. Fox MD, Raichle ME. Spontaneous fluctuations in brain activity observed with functional magnetic resonance imaging. Nat Rev Neurosci 2007;8:700-11 CrossRef Medline

14. Smith SM, Fox PT, Miller KL, et al. Correspondence of the brain's functional architecture during activation and rest. Proc Natl Acad Sci U S A 2009;106:13040 - 45 CrossRef Medline

15. Thompson DK, Inder TE, Faggian N, et al. Corpus callosum alterations in very preterm infants: perinatal correlates and 2 year neurodevelopmental outcomes. Neuroimage 2012;59:3571-81 CrossRef Medline

16. Smyser CD, Inder TE, Shimony JS, et al. Longitudinal analysis of neural network development in preterm infants. Cereb Cortex 2010; 20:2852-62 CrossRef Medline

17. Raichle ME, MacLeod AM, Snyder AZ, et al. A default mode of brain function. Proc Natl Acad Sci U S A 2001;98:676-82 CrossRef Medline

18. Palacios EM, Sala-Llonch R, Junque $C$, et al. Resting-state functional magnetic resonance imaging activity and connectivity and cognitive outcome in traumatic brain injury. JAMA Neurol 2013;70: 845-51 CrossRef Medline

19. Buckner RL, Andrews-Hanna JR, Schacter DL. The brain's default network: anatomy, function, and relevance to disease. Ann NY Acad Sci 2008;1124:1-38 CrossRef Medline

20. Hagmann P, Cammoun L, Gigandet X, et al. Mapping the structural core of human cerebral cortex. PLoS Boil 2008;6:e159 CrossRef Medline

21. Basser PJ, Mattiello J, LeBihan D. MR diffusion tensor spectroscopy and imaging. Biophys J 1994;66:259-67 CrossRef Medline

22. Hagmann $\mathrm{P}$, Jonasson L, Maeder $\mathrm{P}$, et al. Understanding diffusion MR imaging techniques: from scalar diffusion-weighted imaging to diffusion tensor imaging and beyond. Radiographics 2006;26(suppl 1):S205-23 Medline

23. Beaulieu C. The basis of anisotropic water diffusion in the nervous system: a technical review. NMR Biomed 2002;15:435-55 CrossRef Medline

24. Schmithorst VJ, Yuan W. White matter development during adolescence as shown by diffusion MRI. Brain Cogn 2010;72:16-25 CrossRef Medline

25. Jenkinson M, Bannister P, Brady M, et al. Improved optimization for the robust and accurate linear registration and motion correction of brain images. Neuroimage 2002;17:825-41 CrossRef Medline

26. Smith SM. Fast robust automated brain extraction. Hum Brain Mapp 2002;17:143-55 CrossRef Medline

27. Beckmann CF, Smith SM. Probabilistic independent component analysis for functional magnetic resonance imaging. IEEE Trans Med Imaging 2004;23:137-52 CrossRef Medline

28. Smith SM, Jenkinson M, Woolrich MW, et al. Advances in functional and structural MR image analysis and implementation as FSL. Neuroimage 2004;23(suppl 1):S208-19 CrossRef Medline

29. Chen Y, Tymofiyeva O, Hess CP, et al. Effects of rejecting diffusion directions on tensor-derived parameters. Neuroimage 2015;109: 160-70 CrossRef Medline

30. Behrens TE, Berg HJ, Jbabdi S, et al. Probabilistic diffusion tractography with multiple fibre orientations: what can we gain? Neuroimage 2007;34:144-55 CrossRef Medline

31. Johansen-Berg H, Della-Maggiore V, Behrens TE, et al. Integrity of white matter in the corpus callosum correlates with bimanual co-ordination skills. Neuroimage 2007;36(suppl 2):T16-21 CrossRef Medline

32. Mayer KM, Vuong QC. TBSS and probabilistic tractography reveal white matter connections for attention to object features. Brain Struct Funct 2014;219:2159-71 CrossRef Medline

33. Cui J, Tkachenko O, Gogel H, et al. Microstructure of frontoparietal connections predicts individual resistance to sleep deprivation. Neuroimage 2015;106:123-33 CrossRef Medline

34. Uddin LQ, Kelly AM, Biswal BB, et al. Functional connectivity of default mode network components: correlation, anticorrelation, and causality. Hum Brain Mapp 2009;30:625-37 CrossRef Medline

35. Lou HC, Luber B, Crupain M, et al. Parietal cortex and representation of the mental self. Proc Natl Acad Sci U S A 2004;101:6827-32 CrossRef Medline

36. Counsell SJ, Allsop JM, Harrison MC, et al. Diffusion-weighted imaging of the brain in preterm infants with focal and diffuse white matter abnormality. Pediatrics 2003;112:1-7 CrossRef Medline

37. Thompson DK, Lee KJ, Egan GF, et al. Regional white matter microstructure in very preterm infants: predictors and 7 year outcomes. Cortex 2014;52:60-74 CrossRef Medline

38. Morita T, Morimoto M, Yamada K, et al. Low-grade intraventricular hemorrhage disrupts cerebellar white matter in preterm infants: evidence from diffusion tensor imaging. Neuroradiology 2015;57: 507-14 CrossRef Medline

39. Partridge SC, Mukherjee P, Henry RG, et al. Diffusion tensor imaging: serial quantitation of white matter tract maturity in premature newborns. Neuroimage 2004;22:1302-14 CrossRef Medline

40. van Kooij BJ, de Vries LS, Ball G, et al. Neonatal tract-based spatial statistics findings and outcome in preterm infants. AJNR Am J Neuroradiol 2012;33:188-94 CrossRef Medline

41. Berman JI, Mukherjee P, Partridge SC, et al. Quantitative diffusion tensor MRI fiber tractography of sensorimotor white matter development in premature infants. Neuroimage 2005;27:862-71 CrossRef Medline

42. Hüppi PS, Murphy B, Maier SE, et al. Microstructural brain development after perinatal cerebral white matter injury assessed by diffusion tensor magnetic resonance imaging. Pediatrics 2001;107: 455-60 CrossRef Medline

43. Miller SP, Vigneron DB, Henry RG, et al. Serial quantitative diffusion tensor MRI of the premature brain: development in newborns with and without injury. J Magn Reson Imaging 2002;16:621-32 CrossRef Medline

44. Smyser C, Lambeth J. Perinatal factors affecting development of neural networks in preterm infants. In: Proceedings of the Annual Meeting of the Pediatric Academic Societies, Vancouver, Canada, May 1-4, 2010: E-PAS2010 2010:4150 